International Conference Mathematical and Computational Biology 2011

International Journal of Modern Physics: Conference Series

Vol. 9 (2012) 119-126

(C) World Scientific Publishing Company

DOI: $10.1142 / \mathrm{S} 201019451200517 \mathrm{X}$

\title{
NUMERICAL INVESTIGATION OF THE RELEASE OF DRUG FROM A DRUG-DELIVERY DEVICE
}

\author{
M. GARSHASBI \\ School of Mathematics \& Computer Sciences, \\ Damghan University, Damghan, Iran \\ garshasbi@du.ac.ir \\ H. KAMAL GHARIBI \\ School of Mathematics \& Computer Sciences, \\ Damghan University, Damghan, Iran
}

\begin{abstract}
In this study a mathematical model for the release of drug from porous, nonswelling transdermal drug-delivery devices to a perfect sink is considered. The drug is postulated to diffuse in both the solvent-filled pores and the body of the polymer. The limit in which the dissolution of drug is the dominant process is considered and a moving boundary problem governing drug release is derived. This model is solved numerically under some spacial conditions. Furthermore, another limit case in which the dissolution process is not so rapid is investigated and analytical and numerical solutions based on Fourier series solution and finite differences method are derived. Finally, the numerical and analytical solutions are compared to show the ability of the proposed numerical procedures.
\end{abstract}

Keywords: Drug-delivery devices; mathematical model; moving boundary problem.

PACS numbers: 02.60.Lj, 02.70.Bf

\section{Introduction}

In the last 100 years, drug delivery systems have enormously increased their performances, moving from simple pills to sustained/controlled release and sophisticated programmable delivery systems. Transdermal drug delivery system (TDDS) established itself as an integral part of novel drug delivery systems. It is the non-invasive delivery of medications from the surface of skin-the largest and most accessible organ of human body- through its layers, to the circulatory system [1].

The release of a drug from a delivery system involves factors of both dissolution and diffusion. Dissolution rate influences drug release and diffusion cause the transport of the drugs from dosage matrices and into the body [2]

The first example of a mathematical model which describes drug release from a matrix system has been proposed by Higuchi in 1961 [3]. Higuchi developed an equation for the release of a drug from an ointment base and applied it to diffusion 
of solid drugs dispersed in homogenous and granular matrix dosage-systems. Some authors consider the mathematical models of delivery devices in which diffusion occurs solely in the solvent-filled pores or solely in the body of a nonporous polymer. These models have been presented in the cases of membrane devices $[6,7]$ and matrix devices loaded above [4-9] or below the solubility of the drug in the device $[10,11]$. Some models which consider the binding of drug to the polymer, as well as diffusion in the pores, have also been studied [12]. Discrepancies between the models and experimental data have often been attributed to the possible simultaneous diffusion within the two domains of the porous device $[5,13]$. A. J. Lee and his co-workers [13], have been presented a general model for the release of drug from porous, nonswelling transdermal drug-delivery devices to a perfect sink.

This paper is organized as follows:

In section 2 , the mathematical models of the release of drug from drug-delivery devices to a perfect sink in limit cases are presented. Section 3 contains some numerical procedures of the proposed problems obtained in section 2 . In section 4 the numerical and analytical solutions for some examples are compared.

\section{Mathematical Model}

In this section we consider a drug delivery system that takes the form of a patch which contains a certain configuration of matrix devices consist of an impermeable backing and a polymer matrix which contains the drug. The drug is postulated to diffuse in both the solvent-filled pores and the body of the polymer. By considering two limits in which the dissolution of drug is rapid or not, different problems are obtained. The transfer of dissolved drug between the polymer and the solvent (resident in the pores of the device) is considered to be a reversible interconversion process with associated rate constants, $K_{C}$ and $K_{B}$. The concentration of drug dissolved in the pores of the device is denoted by $C(z, t)$, that of dissolved drug in the body of the polymer by $B(z, t)$, and that of undissolved (or dispersed) drug in the pores by $C_{u}(z, t)$. We assume no dispersed drug resident in the polymer.

\subsection{Large dissolution rate limit}

Here we consider that the dissolution rate is large and drug lost due to diffusion or interconversion is quickly replaced by drug coming into solution. Osmotic effects due to solvent already resident in the pores or due to ingress of solvent are assumed to be negligible, and the pores are considered to contain no air. In the case where release occurs only via solvent-filled pores and no drug enters the body of the polymer, following problem may be derived [13]

$$
\begin{aligned}
& \frac{\partial C_{u}}{\partial t}=0, \quad-1<x<-s(t), \\
& \frac{\partial C}{\partial t}=\frac{\partial^{2} C}{\partial x^{2}}, \quad-s(t)<x<0,
\end{aligned}
$$




$$
\begin{gathered}
C\left((-s)^{+}, t\right)=1, \quad \frac{\partial C}{\partial t}\left((-s)^{+}, t\right)=-\dot{s} L c, \quad x=-s(t), \\
s=0, \quad C_{u}=L_{c} \quad t=0, \\
C(x, 0)=1 .
\end{gathered}
$$

The analytic solution to the problem is

$$
c(\chi)=\frac{1}{\operatorname{erf}(-\alpha)} \operatorname{erf}(\alpha \chi), \quad s(t)=2 \alpha \sqrt{t}
$$

where $\operatorname{erf}$ denotes the error function and $\alpha$ is the solution of the transcendental equation

$$
\alpha=\frac{\exp \left(-\alpha^{2}\right)}{\pi L_{c} \operatorname{erf}(-\alpha)}
$$

\subsection{The saturated-reservoir limit}

Some times the dissolution process may not be so rapid [14]. In one special case in which the dispersed drug forms a saturated reservoir for the duration of application $t^{*}$, that is, when $C_{u}, B_{u}>0$ for $0<t<t^{*}$, This situation results the following system of equation

$$
\begin{aligned}
& \frac{\partial C}{\partial t}=\frac{\partial^{2} C}{\partial x^{2}}+K_{C}(1-C)+k_{C}(B-C), \\
& \frac{\partial B}{\partial t}=R \frac{\partial^{2} B}{\partial x^{2}}+K_{B}(1-B)+k_{B}(C-B),
\end{aligned}
$$

where $K_{C}$ and $K_{B}$ are the dissolution rate constants in the pores and the body of polymer respectively. In addition following initial and boundary conditions are at hand

$$
\begin{aligned}
& \text { at } t=0: \quad C=1, \quad B=1 \text {, } \\
& \text { at } \quad x=0: \quad \frac{\partial C}{\partial x}=0, \quad \frac{\partial B}{\partial x}=0, \\
& \text { at } x=1: \quad C=0, \quad B=0 .
\end{aligned}
$$

The system of equations (8) is linear and can be solved analytically. Using Fourier series to solve of equations yields

$$
\begin{aligned}
& C(x, t)=\sum_{n=0}^{\infty} G_{n}(t) \cos \lambda_{n} x, \\
& B(x, t)=\sum_{n=0}^{\infty} F_{n}(t) \cos \lambda_{n} x
\end{aligned}
$$

where $\lambda_{n}=\cos \frac{(2 n+1)}{2} \pi$ and

$$
G_{n}(t)=-c_{1} A \exp \left(\lambda_{1} t\right)-c_{2} D \exp \left(\lambda_{2} t\right), F_{n}(t)=c_{1} \exp \left(\lambda_{1} t\right)+c_{2} \exp \left(\lambda_{2} t\right),
$$

where

$$
A=\frac{\left(k_{B}+K_{B}-K_{c}\right)+R \lambda_{n}^{2}+\lambda_{1}}{\left(k_{c}+K_{c}-K_{B}\right)+\lambda_{n}^{2}+\lambda_{1}}, D=\frac{\left(k_{B}+K_{B}-K_{c}\right)+R \lambda_{n}^{2}+\lambda_{2}}{\left(k_{c}+K_{c}-K_{B}\right)+\lambda_{n}^{2}+\lambda_{2}},
$$


and $\lambda_{1}$ and $\lambda_{2}$ are the roots of following equation

$$
\lambda^{2}+(K) \lambda+M=0,
$$

where

$$
K=k_{c}+K_{c}+k_{B}+K_{B}+\lambda_{n}^{2}+R \lambda_{n}^{2},
$$

and

$$
M=\left(k_{c}+K_{c}+\lambda_{n}^{2}\right)\left(k_{B}+K_{B}+R \lambda_{n}^{2}\right)-2 .
$$

Furthermore the parameters $c_{1}$ and $c_{2}$ can be determined by using initial conditions.

\section{Numerical Solution}

\subsection{Boundary immobilization method}

In this section a numerical procedure based on finite difference method is introduced to solve the proposed Stefan problem. We employ an implicit finite difference method. The moving boundary-value problem governed by equations (1)-(5) can be transformed into a fixed one by means of the mapping $(x, t) \rightarrow(\chi, t)$, where $\chi=\frac{x}{s(t)}$. Using this mapping yields

$$
\begin{gathered}
c_{u}=L_{c}, \quad \frac{\partial c}{\partial \chi}=0, \quad \chi=-1 \\
z \frac{\partial c}{\partial t}-\frac{\chi}{2} \frac{\partial z}{\partial t} \frac{\partial c}{\partial \chi}=\frac{\partial^{2} c}{\partial \chi^{2}}, \quad-1<\chi<0 \\
-\frac{\partial c}{\partial t}=\frac{L c}{2} \frac{d z}{d t}, \quad \chi=-1 \\
c=1 \quad t=0, \quad c=0, \quad \chi=0
\end{gathered}
$$

where $z=s^{2} \quad$ and $z\left(t_{0}\right)=0$.

By discretisizing this problem one may have

$$
a_{i}^{(k+1)} c_{i-1}^{(k+1)}+b_{i}^{(k+1)} c_{i}^{(k+1)}+d_{i}^{(k+1)} c_{i+1}^{(k+1)}=z^{(k)} c_{i}^{(k)}
$$

where

$$
\begin{aligned}
& \left.a_{i}^{(k+1)}=r\left(i \frac{(\Delta \chi)^{2}}{4}\left(\frac{\partial z}{\partial t}\right)^{(k)}\right)-1\right), \\
& b_{i}^{(k+1)}=z^{(k)}+2 r, \\
& \left.d_{i}^{(k+1)}=-r\left(i \frac{(\Delta \chi)^{2}}{4}\left(\frac{\partial z}{\partial t}\right)^{(k)}\right)-1\right),
\end{aligned}
$$


which holds for $i=1,2, \ldots, N-1$ and $k=0,1,2, \ldots, L$ with $r=\Delta t / \Delta \chi^{2}$ where $\Delta t$ and $\Delta \chi$ denote the sizes of the temporal and spatial steps, respectively. The Stefan condition in (13) becomes

$$
\left(\frac{\partial z}{\partial t}\right)^{(k)}=\frac{-1}{L c} \frac{3 c_{N}^{(k)}-4 c_{N-1}^{(k)}+c_{N-2}^{(k)}}{\Delta \chi}
$$

and the position of the moving boundary is updated via the formula

$$
z^{(k+1)}=z^{(k)}-\Delta t \frac{1}{L c} \frac{3 c_{N}^{(k)}-4 c_{N-1}^{(k)}+c_{N-2}^{(k)}}{\Delta \chi} .
$$

Also using (6), we have

$$
c_{i}^{i}=\frac{1}{\operatorname{erf}(-\alpha)} \operatorname{erf}\left(\alpha \chi_{i}\right), \quad i=0,1,2, \cdots .
$$

\subsection{Numerical solution of the saturated-reservoir limit model}

In order to implement a numerical approach for solving system of equations (8), in this section two numerical procedures based on finite differences method are proposed. The proposed numerical procedures are explained as follows.

\subsubsection{The implicit finite-difference scheme}

As the first numerical approach, we employ an implicit finite-difference method to discrete equations (8). To this end let $\Delta t=t_{j}-t_{j-1}$ be the time step and $h=x_{i+1}-x_{i}$ be the spatial step,and Let $\mathrm{N}$ be the total number of mesh points in $[0,1]$. Then the discretization form of the system is may be derived as as

$$
\begin{gathered}
(-r) c_{i-1}^{k+1}+\left[1+2 r+\Delta t k_{1}\right] c_{i}^{k+1}-r c_{i+1}^{k+1}-\left(\Delta t k_{c}\right) B_{i}^{k+1}=c_{i}^{k}+\Delta t k_{c}, \\
(-R r) B_{i-1}^{k+1}+\left[1+2 R r+\Delta t k_{2}\right] B_{i}^{k+1}-(R r) B_{i+1}^{k+1}-\left(\Delta t k_{B}\right) c_{i}^{k+1}=B_{i}^{k}+\Delta t k_{B},
\end{gathered}
$$

where

$$
r=\frac{\Delta t}{h^{2}}, \quad k_{1}=k_{c}+K_{c}, \quad k_{2}=k_{B}+K_{B}
$$

and hold for $i=1,2, . ., N-1$

\subsubsection{The Crank-Nicolson scheme}

We now consider the well-known Crank-Nicolson scheme which is unconditionally stable and second-order accurate. The method involves using a central difference at time $t^{n+\frac{1}{2}}$ and a second-order central difference for the space derivative at position 
$x$. We apply the Crank-Nicolson scheme to the system (8). Using Crank-Nicolson method yields

$$
\begin{gathered}
-\frac{r}{2} c_{i-1}^{k+1}+\frac{1}{2}\left(2+2 r+\Delta t k_{1}\right) c_{i}^{k+1}-\frac{r}{2} c_{i+1}^{k+1}-\frac{\Delta t k_{c}}{2} B_{i}^{k+1} \\
=\frac{r}{2} c_{i-1}^{k}+\frac{1}{2}\left(2-2 r-\Delta t k_{1}\right) c_{i}^{k} \frac{r}{2} c_{i+1}^{k}+\frac{\Delta t k_{c}}{2} B_{i}^{k} \\
-\frac{r R}{2} B_{i-1}^{k+1}+\frac{1}{2}\left(2+2 R r+\Delta t k_{2}\right) B_{i}^{k+1}-\frac{R r}{2} B_{i+1}^{k+1}-\frac{\Delta t k_{B}}{2} c_{i}^{k+1} \\
=\frac{R r}{2} B_{i-1}^{k}+\frac{1}{2}\left(2-2 R r-\Delta t k_{1}\right) B_{i}^{k} \frac{R r}{2} B_{i+1}^{k}+\frac{\Delta t k_{B}}{2} c_{i}^{k}
\end{gathered}
$$

where

$$
r=\frac{\Delta t}{h^{2}}, \quad k_{1}=k_{c}+K_{c}, \quad k_{2}=k_{B}+K_{B}
$$

and hold for $i=1,2, . ., N-1$.

\section{Results and Discussion}

In this section for illustrations purpose, the comparison between the numerical and analytical results are discussed. First we consider the problem (1)-(5). Table 1 shows the results obtained by the BIM and the exact solution for $u(x, t)$. This table shows that the results obtained by the present method are in excellent agreement with the analytic results.

Table 1. The comparison between numerical and exact solution; $N=100, \Delta t=10^{-} 3$.

\begin{tabular}{ccc}
\hline$\chi$ & numerical solution & exact solution \\
\hline-.1 & 0.112803319134183 & 0.112803070664921 \\
-.2 & 0.224743100902460 & 0.224742621276906 \\
-.3 & 0.334975588857706 & 0.334974912203066 \\
-.4 & 0.442695835126561 & 0.442695011400216 \\
-.5 & 0.547155261592492 & 0.547154355139492 \\
-.6 & 0.647677118778582 & 0.647676206471148 \\
-.7 & 0.743669316069437 & 0.743668485082386 \\
-.8 & 0.834634231158758 & 0.834633576447717 \\
-.9 & 0.920175256849138 & 0.920174878410277 \\
\hline
\end{tabular}

Table 2 displays the estimation of the interface location when $t_{f}=0.5$. It can be seen the numerical results are in good agreement with the exact one, e.g., at $t_{f}=0.5$. The percentage error decreasing from $2.8 \times 10^{-} 2(N=10)$ to $5.1 \times 10^{-} 3(N=80)$.

Table 3 presents the values obtained by applying Richardson's extrapolation to the value of the weighted 1 -norm defined by

$$
\|e\|_{1}=\frac{1}{N} \sum_{i=0}^{N-1}\left|1-\frac{u_{i}^{m}}{U\left(x_{i}, t_{m}\right)}\right|, \quad e=\left[e_{0} \ldots e_{N-1}\right]^{T} .
$$


Table 2. Convergence analysis for the BIM method $t_{f}=0.5$. Exact solution $=$ -0.876888492468601 .

\begin{tabular}{ccc}
\hline $\mathrm{L}$ & $\mathrm{s}$ & ERROR \\
\hline 10 & -0.877172333579786 & $2.838 \times 10^{-4}$ \\
20 & -0.876982162345392 & $9.366 \times 10^{-5}$ \\
40 & -0.876947433723859 & $5.894 \times 10^{-5}$ \\
80 & -0.876940334927502 & $5.184 \times 10^{-5}$ \\
\hline
\end{tabular}

Table 3. The values of $\|e\|_{1}$ of the numerical solutions.

\begin{tabular}{cc}
\hline $\mathrm{h}$ & $\|e\|_{1}$ \\
\hline-.05 & $2.26 \times 10^{-} 5$ \\
-.025 & $5.93 \times 10^{-} 6$ \\
-.0125 & $1.93 \times 10^{-} 6$ \\
\hline
\end{tabular}

In order to study the permeation of drug in porous matrices the following data are used

$$
k_{c}=1, \quad K_{c}=1, \quad k_{B}=1, \quad K_{B}=2, \quad R=10^{-1} .
$$

Since the diffusivity of drug in the body of the polymer is often considered to be much smaller than that in the solvent-filled pores of the device [4], we shall therefore restrict attention to the case where $\mathrm{R}$ is small. Now by approximating the exact solution for the first 200 sentences of series from (10), we can comparison numerical and analytically results. In the case of saturated-reservoir limit, as reflected in Table 4, the methods employed give good results compared with the analytic solutions.

Table 4. The comparison between numerical and exact solution; $N=100, \Delta t=10^{-} 3$.

\begin{tabular}{lcccc|cc}
\hline $\mathrm{x}$ & \multicolumn{2}{c}{ Implicit } & \multicolumn{2}{c}{ Crank-Nicolson } & \multicolumn{2}{c}{ Exact solution } \\
\cline { 2 - 7 } & $\mathrm{C}$ & $\mathrm{B}$ & $\mathrm{C}$ & $\mathrm{B}$ & $\mathrm{C}$ & $\mathrm{B}$ \\
\hline 0.1 & 0.9451 & 0.9965 & .9490 & 0.9975 & 0.9499 & 0.9977 \\
0.2 & 0.9266 & 0.9949 & 0.9286 & 0.9960 & 0.9297 & 0.9962 \\
0.3 & 0.8937 & 0.9919 & 0.8926 & 0.9932 & 0.8937 & 0.9934 \\
0.4 & 0.8431 & 0.9868 & 0.8381 & 0.9882 & 0.8391 & 0.9885 \\
0.5 & 0.7708 & 0.9788 & 0.7618 & 0.9801 & 0.7627 & 0.9804 \\
0.6 & 0.6730 & 0.9664 & 0.6610 & 0.9675 & 0.6616 & 0.9678 \\
0.7 & 0.5468 & 0.9478 & 0.5337 & 0.9484 & 0.5341 & 0.9487 \\
0.8 & 0.3911 & 0.9206 & 0.3799 & 0.9205 & 0.3801 & 0.9208 \\
0.9 & 0.2074 & 0.8747 & 0.2009 & 0.8734 & 0.2011 & 0.8813 \\
\hline
\end{tabular}




\section{Conclusion}

In this paper the mathematical model of the release of drug from porous, nonswelling transdermal drug-delivery devices to a perfect sink has been considered in two special cases. The drug has been postulated to diffuse in both the solvent-filled pores and the body of the polymer. The mathematical models have been derived as a moving boundary value problem and a system of parabolic partial differential equations. The proposed models have been investigated numerically and the numerical results have shown a good agreement with the analytic results.

\section{References}

1. O. Pillai, A. B. Dhanikula and R. Panchagnula, Current Opinion in Chemical Biology. 5, 439 (2001).

2. N. Alfred and J. Patrick, Martins physical pharmacy and pharmaceutical sciences, 6th edn. (Piscataway, New Jersey, California Institute of Technology, 2009).

3. T. Higuchi, Journal pharmaceutical Science. 50, 874 (1961).

4. N. A. Peppas, J. Biomed. Mater. Res. 17, 1079 (1983).

5. N. A. Peppas, Medical Applications of Controlled Release. Vol. II. Boca Raton, FL: CRC Press (1984).

6. A. Gopferich and G. Lee, Prediction of Percutaneous Penetration, eds. R. C. Scott and R. H. Guy (London: IBC Technical Services 1991).

7. G. Gienger, A. Knoch and H. P. Merkle, J. Pharm. Sci. 75, 9 (1986).

8. T. Higuchi, J. Pharm. Sci. SI. 52, 1145 (1963).

9. J. W. Ayres and F. T. Linstrom, Pharm. Sci. 66, 654 (1977).

10. A. Gopferich and G. Lee, Int. J. Pharm. 71, 237 (1991).

11. W. J. Addicks, G. Flynn, N. Weiner and R. Curl, Int. J. Pharm. 56, 243 (1989).

12. S. J. Desal, P. Singh, A. P. Simonelli and W. I. Higuchi, J. Pharm. Sci. 55, 1224 (1966).

13. A. J. Lee, J. R. King and S. Hibberd, IMA J. Math. Appl. Med. EJ Bio. 15, 135 (1998).

14. R. A. Siegal, Modelling of drug release from porous polymers. In: Controlled Release of Drugs: Polymers and Aggregate Systems (New York: VCH. 1989). 